\title{
Top-down analysis of 30-80 kDa proteins by electron transfer dissociation time-of-flight mass spectrometry
}

\author{
Luca Fornelli • Julien Parra • Ralf Hartmer • \\ Carsten Stoermer • Markus Lubeck • Yury O. Tsybin
}

Received: 2 June 2013 / Revised: 16 July 2013 / Accepted: 17 July 2013 / Published online: 10 August 2013

(C) Springer-Verlag Berlin Heidelberg 2013

\begin{abstract}
Electron transfer dissociation (ETD)-based topdown mass spectrometry (MS) is the method of choice for in-depth structure characterization of large peptides, smalland medium-sized proteins, and non-covalent protein complexes. Here, we describe the performance of this approach for structural analysis of intact proteins as large as the $80 \mathrm{kDa}$ serotransferrin. Current time-of-flight (TOF) MS technologies ensure adequate resolution and mass accuracy to simultaneously analyze intact $30-80 \mathrm{kDa}$ protein ions and the complex mixture of their ETD product ions. Here, we show that ETD TOF MS is efficient and may provide extensive sequence information for unfolded and highly charged (around 1 charge/ $\mathrm{kDa}$ ) proteins of $\sim 30 \mathrm{kDa}$ and structural motifs embedded in larger proteins. Sequence regions protected by disulfide bonds within intact non-reduced proteins oftentimes remain uncharacterized due to the low efficiency of their fragmentation by ETD. For serotransferrin, reduction of S-S bonds leads to significantly varied ETD fragmentation pattern with higher sequence coverage of $\mathrm{N}$ - and C-terminal regions, providing a complementary structural information to topdown analysis of its oxidized form.
\end{abstract}

Electronic supplementary material The online version of this article (doi:10.1007/s00216-013-7267-5) contains supplementary material, which is available to authorized users.

\footnotetext{
L. Fornelli $\cdot$ J. Parra $\cdot$ Y. O. Tsybin $(\bowtie)$

Biomolecular Mass Spectrometry Laboratory, Ecole Polytechnique

Fédérale de Lausanne, 1015 Lausanne, Switzerland

e-mail: yury.tsybin@epfl.ch

R. Hartmer $\cdot$ C. Stoermer $\cdot$ M. Lubeck

Bruker Daltonics GmbH, Fahrenheitstraße 4, 28359 Bremen,

Germany

Present Address:

J. Parra

Laboratory for Analysis and Modelling for Biology and Environment, CNRS UMR 8587, Université d'Evry Val d'Esonne, 91025 Evry Cedex, France
}

Keywords Electron transfer dissociation $\cdot$ ETD . Electrospray $\cdot$ ESI $\cdot$ Tandem MS $\cdot$ MS/MS $\cdot$ Time-of-flight mass spectrometry $\cdot$ TOF MS $\cdot$ Top-down $\cdot$ Transferrin

\section{Introduction}

The term top-down mass spectrometry (TD MS) refers to the structure analysis of intact protein ions by their fragmentation in the gas phase [1]. When applied to a mixture of proteins from different protein families or proteoforms of a single protein family, TD MS translates into a top-down proteomics (TDP). TDP presents several theoretical advantages over bottom-up mass spectrometry-based proteomics (BUP), which consists in the analysis of peptide mixtures derived from proteolysis [2, 3]. In both TDP and BUP, the survey scan (MS ${ }^{1}$ or simply MS) yields information about the total mass of biomolecules, whereas the tandem MS (MS/MS) step confirms the sequence of the selected precursor ion. TDP thus identifies a specific proteoform, whereas BUP is typically employed in identification of protein families [4].

TD MS and TDP analyses are technically more challenging since proteins are generally more difficult to separate, ionize, and fragment than peptides. In addition, the resulting MS/MS spectra are convoluted and data interpretation is not trivial. Recent introduction of novel in-solution protein fractionation techniques, e.g., gel-eluted liquid fraction entrapment electrophoresis [5], or the adoption of new stationary phases for liquid chromatography (LC) [6] have partially filled the gap between peptide and protein fractionation and separation. However, for MS data acquisition and analysis, new developments and improvements are needed. In terms of hardware, only high-resolution instruments are capable of resolving and distinguishing complex product ion clusters in MS/MS spectra of proteins. In terms of software, specific data analysis workflows have to be applied to efficiently reconstruct and 
combine the information from $\mathrm{MS}^{1}$ and, most importantly, MS/MS.

Although all the common ion activation methods can be applied to intact proteins, electron-based fragmentations, specifically electron capture dissociation (ECD) $[7,8]$ and electron transfer dissociation (ETD) [9], have been shown to produce a higher sequence coverage than "slow-heating" methods (such as collision-induced dissociation [10] or infrared multiphoton dissociation [11]) and also have the capability of cleaving disulfide bridges [12]. Importantly, ECD and ETD perform most efficiently (i.e., with low ion activation time resulting in an extensive backbone fragmentation) with highly charged precursors, which is the typical condition present during TD MS or TDP where highly charged protein cations are analyzed. Recently, developments in ultraviolet photodissociation (UVPD) and surface-induced dissociation MS/MS demonstrated intriguing capabilities specifically for intact protein analysis [13].

Originally, radical-driven TD MS of large proteins was introduced using MS/MS "in time" because the required high resolution was only available for Fourier transform ion cyclotron resonance mass spectrometers (FT-ICR MS) and the ability to perform radical-induced fragmentation was only available in ion traps, e.g., ICR ion trap or Paul ion trap [14, $15]$. Beginning with comprehensive analysis of $\sim 10-\mathrm{kDa}$ model proteins, such as ubiquitin [16], the application range of ECD-based TD MS was significantly expanded, delivering a single-amino acid resolved structure of a $29 \mathrm{kDa}$ carbonic anhydrase $[17,18]$ and further analysis of proteins in the 50 $70 \mathrm{kDa}$ range [19]. TD MS of proteins in these mass ranges can be now performed on the time scale of on-line LC.

The quest for radical-driven fragmentation in the widespread, low resolution quadrupole $(q)$ mass analyzers and product ion analysis in the high-resolution mass analyzers led to the development of radical-driven top-down mass spectrometry "in space". After numerous attempts, ECD was efficiently implemented in a quadrupole mass analyzer that could be coupled to a higher resolution time-of-flight mass spectrometer (TOF MS) [20, 21]. However, the real revolution in TD MS "in space" was made by implementing ETD first in a linear ion trap and then in other quadrupole mass analyzers [22]. Currently, the most successful implementations of ETD specifically for protein analysis include ETD on hybrid qTOF MS [23, 24], LTQ Orbitrap FTMS [25] and qFT-ICR MS platforms [26]. These recent technologies have already shown strong potential applications, including the structural analysis of $\sim 150 \mathrm{kDa}$ intact immunoglobulins $\mathrm{G}$ ( $\mathrm{IgG}$ ) on different platforms [27, 28].

The ETD fragmentation and subsequent mass analysis using a qTOF MS of bovine carbonic anhydrase and human serotransferrin are presented herein. Carbonic anhydrase was used as a model for medium-size proteins, which can be analyzed without specifically designed sample preparation or instrumental set-up. Serotransferrin was used to challenge the instrument capabilities with a large protein and investigate the performance of ion manipulation, fragmentation, and detection. Structural study of this protein is complicated in part because its large MW and the presence of 19 disulfide bridges hinders ion-ion interactions in ETD. This structural complexity manifested itself by significant differences in the ETD fragmentation patterns depending on serotransferrin oxidation state. To characterize these proteins in detail we applied dedicated data analysis workflow based on the SNAP2 algorithm (Bruker Daltonics, Bremen, Germany). Our results thus demonstrate the current capabilities of high-resolution qTOF MS in sequence analysis of large proteins by TD MS.

\section{Experimental}

Sample preparation Bovine carbonic anhydrase and human serum transferrin (serotransferrin) were obtained commercially (Sigma, Taufkirchen, Germany). For direct infusion experiment of carbonic anhydrase and non-reduced serotransferrin, proteins were dissolved in water/acetonitrile/formic acid (50:49.9:0.1, $v / v / v)$ to a final concentration of $\sim 1 \mu \mathrm{M}$ and used without further purification. Transferrin reduction was obtained by dissolving the lyophilized protein in $50 \mathrm{mM}$ sodium carbonate buffer in presence of $5 \mathrm{mM}$ tris(2-carboxyethyl)phosphine (TCEP, Sigma). Prior to infusion, the reduced protein was desalted by centrifugation with microcentrifuge $10 \mathrm{kDa}$ MW cut-off filters (Millipore, Zug, Switzerland).

Time-of-flight tandem mass spectrometry Mass measurements of intact protein ions and ETD MS/MS were performed on a high-resolution, high-mass accuracy quadrupole time-offlight (qTOF) mass spectrometer (maXis UHR qTOF MS, Bruker Daltonics GmbH, Bremen, Germany) equipped with electrospray ion source. ETD MS/MS was performed as previously described [24]. The main steps in top-down ETD TOF MS are schematically shown in Fig. 1. The differences in ETD of high-mass proteins compared to smaller analytes are: (1) prolonged cation accumulation and radical anion injection times to ensure the accumulation of a sufficient number of analyte ions and, at the same time, the possibility of efficiently inducing ETD; (2) decreased ion-ion reaction time as ETD proceeds faster when highly charged ions are used as precursors. After optimization (see Supplementary Information), precursor ion accumulation time was increased up to $800 \mathrm{~ms}$, anion injection time was set to $100 \mathrm{~ms}$, and additional ionion interaction time to $40-50 \mathrm{~ms}$. Product ions were transferred to the ion cooler cell from the reaction cell and orthogonally pushed to the TOF mass analyzer at the rate of $10 \mathrm{kHz}$. The orthogonal acceleration potential was at $10 \mathrm{kV}$. Acquisition in a broad $\mathrm{m} / \mathrm{z}$ window $(100-5,000 \mathrm{~m} / \mathrm{z})$ was performed with signal digitization at $2 \mathrm{GHz}$, whereas higher resolution MS and MS/ 


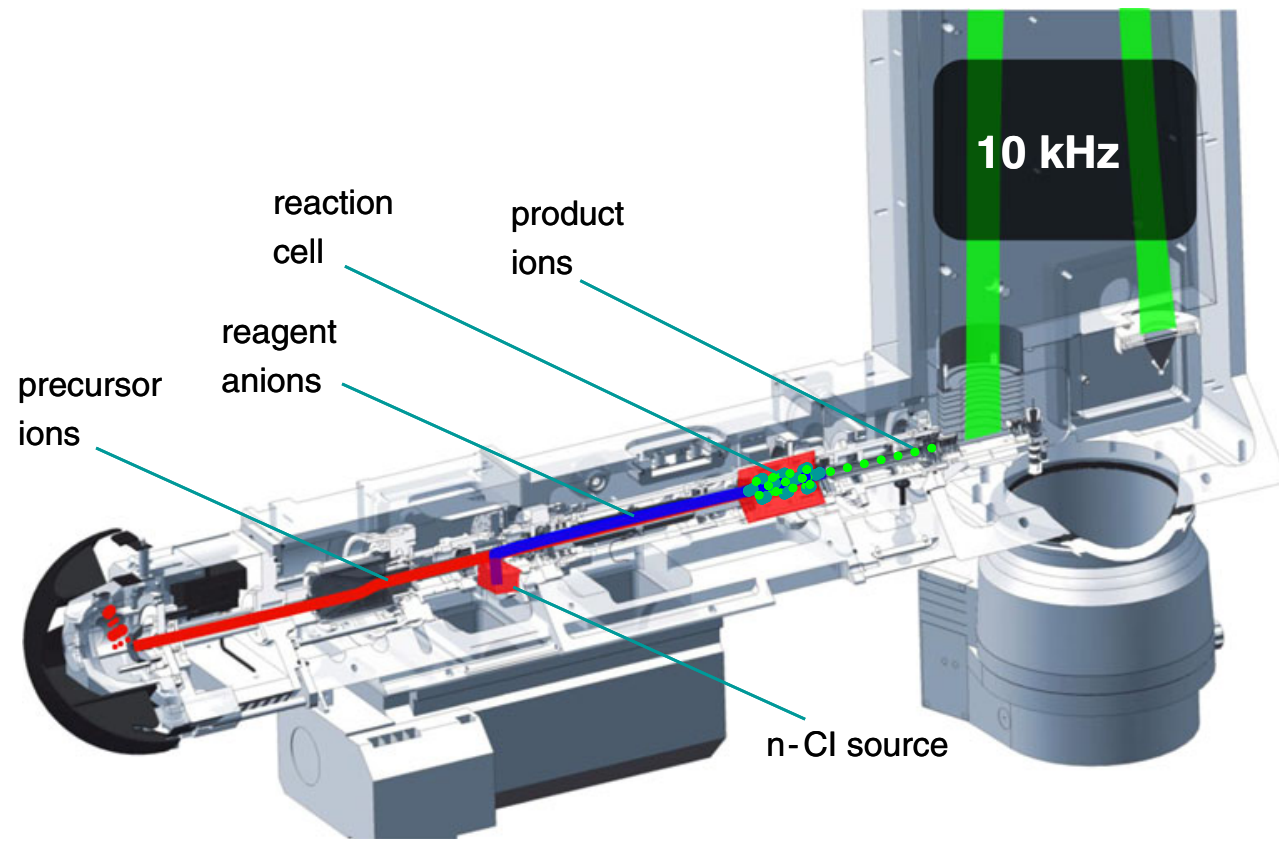

Fig. 1 Sequence of top-down electron transfer dissociation tandem mass spectrometry on a qTOF MS. Step 1: electrospray ionization of the samples in the ion source and precursor ion accumulation in the reaction cell $(800 \mathrm{~ms})$. Step 2: ETD reagent injection from the chemical ionization source to the reaction cell $(20-100 \mathrm{~ms})$. Step 3: ion-ion reaction in the reaction cell $(20-100 \mathrm{~ms})$. Step 4: ion-ion reaction product ions transfer

MS data were acquired at a faster digitization rate of $4 \mathrm{GHz}$ for narrower $\mathrm{m} / \mathrm{z}$ range of $500-3,000 \mathrm{~m} / \mathrm{z}$. Intact protein and product ion mass spectra were acquired in separate direct infusion experiments. For the larger protein, data acquisition time of up to $30 \mathrm{~min}$ was employed to average up to $1,000 \mathrm{MS} / \mathrm{MS}$ spectra.

Data analysis The MS and MS/MS spectra were analyzed using the dedicated top-down data analysis procedure. Briefly, precursor and product ion mass spectra were summed over the infusion time in each MS and MS/MS experiment with Data Analysis software version 4.0 (Bruker Daltonics). Intact protein mass spectra were submitted to the MaxEntropy deconvolution procedure to yield heterogeneous distribution of neutral (deprotonated) glycosylated proteoforms.

Before ETD data analysis, the SNAP 2 algorithm was used to simulate MS/MS spectra with theoretical isotopic distributions of the peaks that matched the experimental mass spectra with a correlation coefficient of 0.4 or better. Charge states and monoisotopic masses were assigned automatically for the product ion isotopic distributions in both the experimental and theoretical mass spectra. Manual analysis considering both experimental and theoretical mass spectra was performed to assign ETD-specific $c$-type and $z$-type product ions. Alternatively, automatic data analysis was performed by deisotoping the simulated isotopic clusters, keeping only the monoisotopic masses. Following charge state deconvolution, the obtained mass list of into the ion cooler. Step 5: ion extraction from the ion cooler into the orthogonal acceleration region. Step 6: orthogonal ion extraction into the time-of-flight tube with an iron mirror $(10 \mathrm{kHz}, 10 \mathrm{kV})$. Step 7: ion detection at the multichannel plate with time to digital converter ( 2 or $4 \mathrm{GHz}$ sampling rate)

product ions was searched against the known sequence of the proteins to determine the product ion identity and the sequence coverage. The results of both manual and automatic data analysis were compared and pooled to generate the verified protein sequence coverage map. Internal product ions, formed by secondary fragmentation of the primary product ions, have not been considered in the current work.

\section{Results and discussion}

Evaluation of ETD top-down mass spectrometry workflow The complete workflow for TD MS includes: (1) sample preparation, (2) intact protein mass measurement in MS mode, (3) sequence analysis in MS/MS mode, and (4) data analysis. The present work employed a state-of-the-art, ETD-enabled highresolution qTOF mass spectrometer (Fig. 1). Some of its figures of merits for intact protein analysis are: resolution up to 50,000 over a wide $\mathrm{m} / \mathrm{z}$ range in $\mathrm{MS}^{1}$, and up to 30,000 40,000 for MS/MS (the resolution is influenced by the complexity and the abundance of the product ion population); a scan rate up to $20 \mathrm{~Hz}$; mass accuracy of $\sim 1 \mathrm{ppm}$ (for both $\mathrm{MS}^{1}$ and MS/MS). Remarkably, TOF mass analyzers generally have a higher dynamic range than FT-based instruments, and this characteristic is particularly important for intact protein analysis: for the $\sim 9-\mathrm{kDa}$ ubiquitin the ratio between the monoisotopic and the most abundant peaks is $\sim 10$, but the same 
ratio increases to $\sim 6 \mathrm{E} 4$ for $\sim 29 \mathrm{kDa}$ carbonic anhydrase, $\sim \mathrm{E} 15$ for $\sim 79 \mathrm{kDa}$ transferrin, and up to $\sim \mathrm{E} 32$ for a $\sim 150-\mathrm{kDa}$ IgG.

Figure 2 shows the ETD mass spectrum of $35^{+}$precursor ion of carbonic anhydrase. Most intense product ions are shown in the expanded segments. Generally, the envelope of the undissociated charge-reduced species (also referred to as "ETnoD products") in ETD TD MS of small to medium proteins is positioned in the low $\mathrm{m} / \mathrm{z}$ range $(\sim 500-1,500 \mathrm{~m} / \mathrm{z})$ therefore overlapping with typical product ions of $~ 300$ $1,400 \mathrm{~m} / \mathrm{z}$. Here, three different intense ETnoD products (with charge states $34^{+}, 33^{+}$, and $32^{+}$) were detected, impeding the product ion observation between $\sim 850$ and $920 \mathrm{~m} / \mathrm{z}$.

The complexity of the product ion mixture for ETD TD MS of carbonic anhydrase is illustrated in Fig. 3, top panel. Several partially overlapping clusters of multiply charged ions can be observed in an expanded 675-684 $\mathrm{m} / \mathrm{z}$ window. Due to the high density of peaks, the first data analysis step is to filter the ion clusters from noise or spurious peaks by matching the experimentally obtained clusters with theoretical peaks (Fig. 3, middle panel). For a successful noise filtration, high-mass accuracy and resolution are needed. In addition, high signal-to-noise signal is required to confidently identify the charge state and possibly the monoisotopic peak of each ion cluster. For carbonic anhydrase, the qTOF TD MS allowed the assignment of product ions of up to $14^{+}$charge state. Software parameters such as signal-to-noise cut-off can be manually selected to enhance peak identifications. The SNAP2 algorithm matches the experimental and theoretical distributions applying a user-defined correlation coefficient. Due to the complexity of product ion population for a $29 \mathrm{kDa}$ protein, this coefficient is usually set to 0.5 or higher (equivalent to $50 \%$ correlation), but this value can be reduced for larger proteins, where generally the complexity of product ion mixture is higher and the average signal-to-noise ratio is lower. Finally, as illustrated in Fig. 3, bottom panel, the identified clusters are deconvoluted and deisotoped, as only the monoisotopic masses are used for the database search or, alternatively, for de novo sequencing. In all our experiments, we imported the sequence of the employed proteins in the software BioTools (Bruker Daltonics) to reduce the time needed for a complete database search.

\section{ETD qTOF MS of Carbonic Anhydrase (29 kDa)}

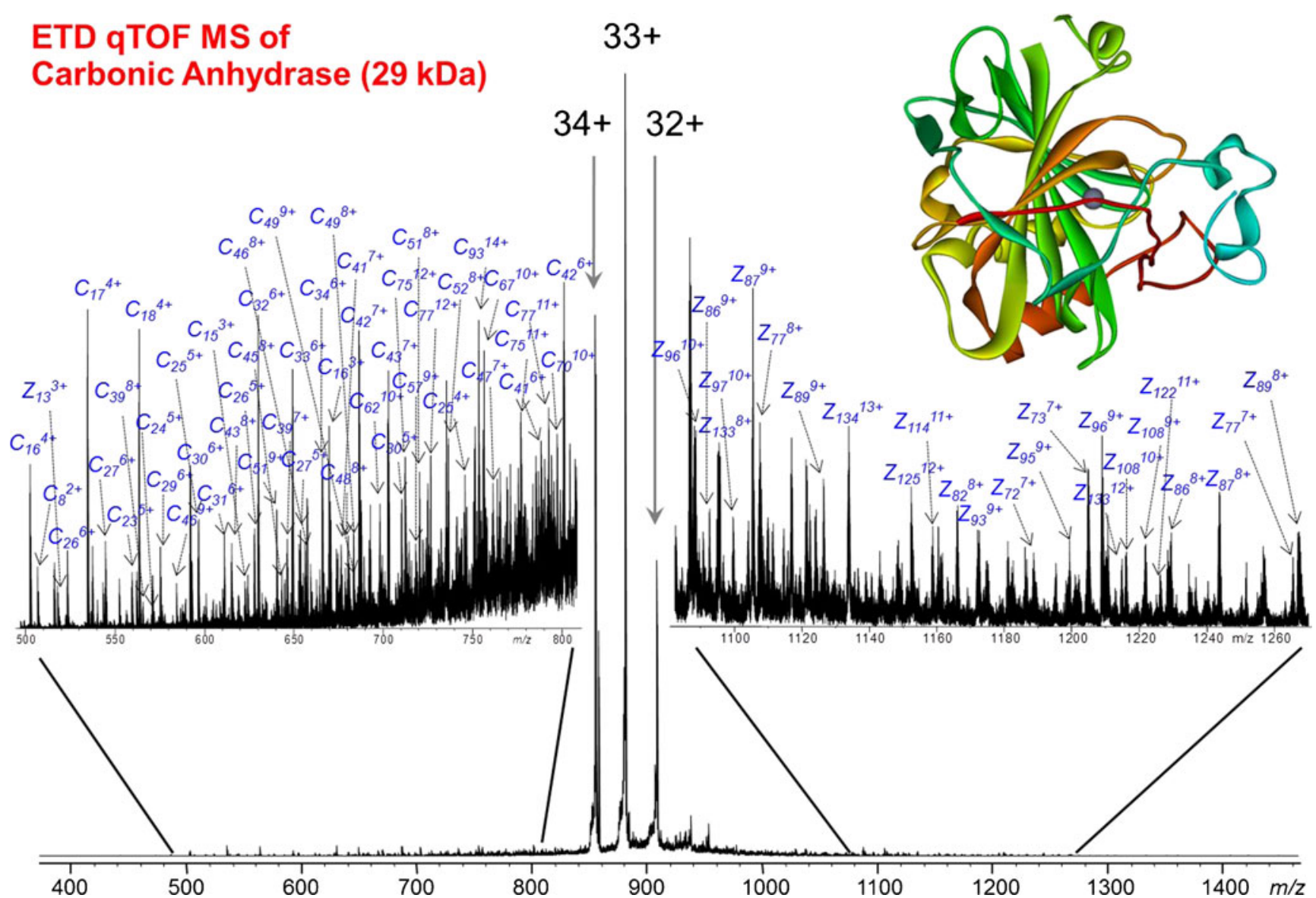

Fig. 2 Top-down electron transfer dissociation (ETD)-based ESI qTOF MS/MS of carbonic anhydrase $(29 \mathrm{kDa})$. Prior to ETD, a single charge state, $[\mathrm{M}+35 \mathrm{H}]^{35+}$, of carbonic anhydrase was selected with an isolation window of $30 \mathrm{~m} / \mathrm{z}$. Efficient charge reduction upon electron transfer resulted in electron transfer products without dissociation (ETnoD products), specifically $[\mathrm{M}+35 \mathrm{H}]^{34+\cdot},[\mathrm{M}+35 \mathrm{H}]^{33+\cdot \cdot}$, and $[\mathrm{M}+35 \mathrm{H}]^{32+\cdots}$ ions. The insets show the expanded regions of the MS/MS mass spectra 
Fig. 3 Top-down MS/MS data analysis procedure. Example is made for ETD qTOF MS/MS of $29 \mathrm{kDa}$ carbonic anhydrase protein, $m / z=675-684$ (Fig. 2).

Step 1: raw data file analysis and matching of theoretical and experimental isotopic clusters. Step 2: deconvolution and deisotoping of the selected theoretical isotopic clusters. Step 3: protein theoretical product ion database search and/or de novo sequencing based on the monoisotopic masses and charge states data (product ion assignment)
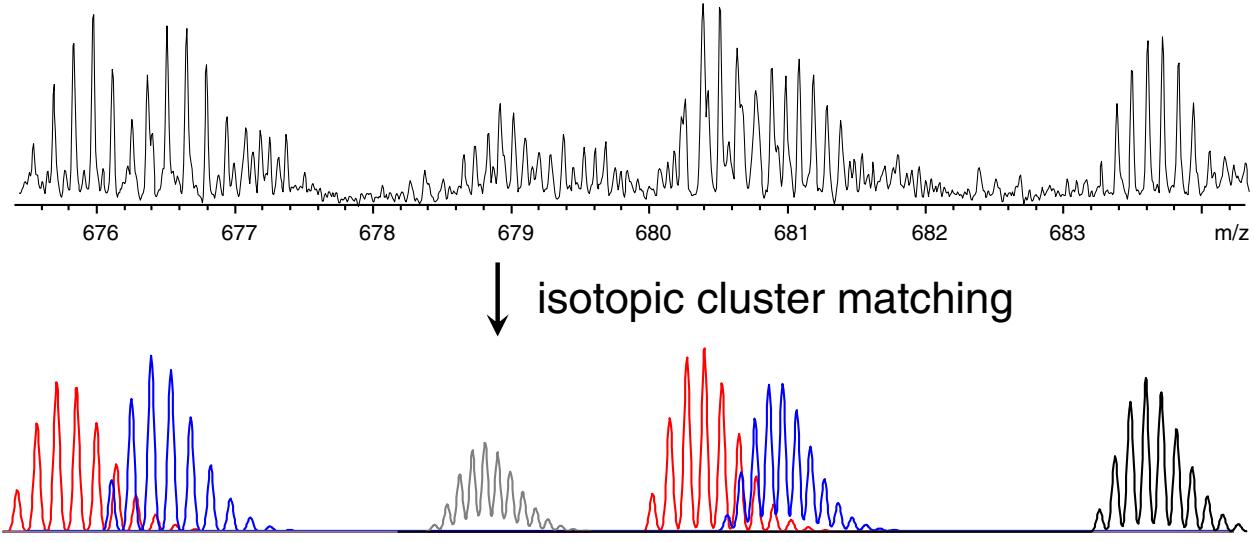

deconvolution and de-isotoping
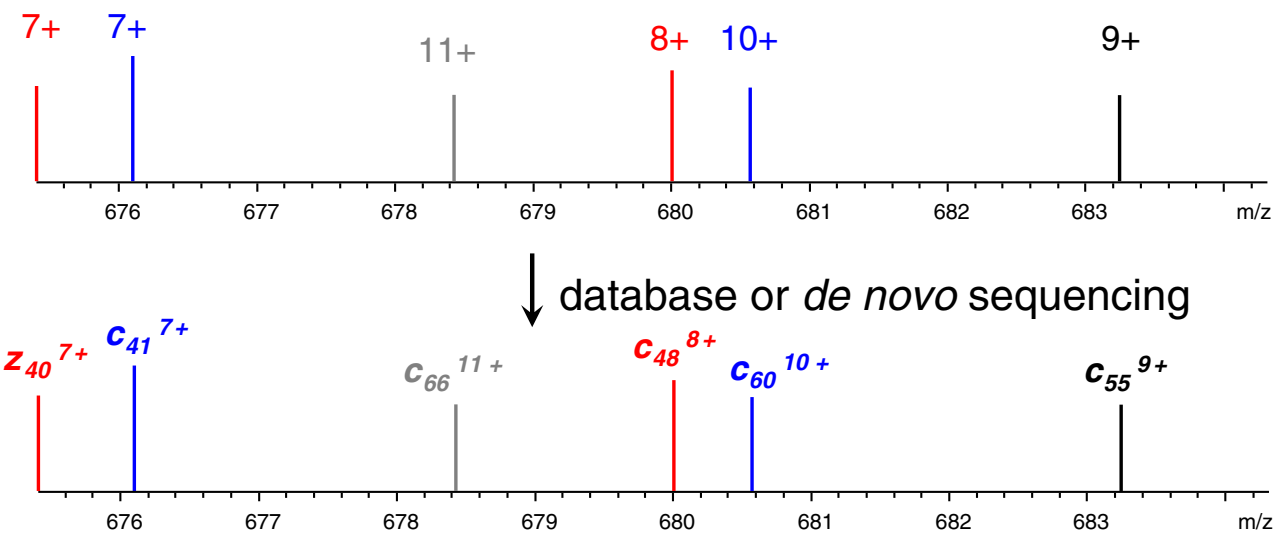

Top-down mass spectrometry of oxidized transferrin Mature human serotransferrin (UniProt entry: P02787) is a 679 amino acid-long protein, Scheme 1. First, the intact mass of non- reduced transferrin was determined (Fig. 4, insets). The observed charge state distribution was centered at $\sim 2,200 \mathrm{~m} / \mathrm{z}$ and included charge states $22-50^{+}$with the most abundant
Scheme 1 Fragmentation map of intact transferrin after ETD-based ESI qTOF MS/MS. Backbone cleavage sites identified uniquely for the oxidized form of serotransferrin are indicated in black, whereas cleavages characteristic of the reduced form are indicated in red. Common cleavage sites are shown in green. Disulfide bridges are indicated by purple dotted lines

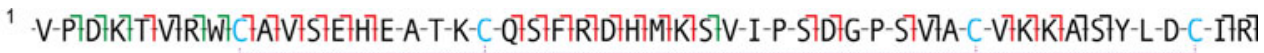

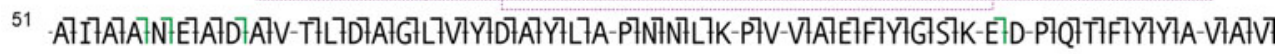
101 VTKTK-D†S-GTFFQ-M-N-Q7L-RTG-K-K-S-C-H-T-G-L-G-R-S-A-G-W-N-I-P-I-G-L-L-Y-C-D-L-P-E-P-R-K-P-L-E-K-A-V. 151 A-N-F-F-S-G-S-C-A-P-C-A-D-G-T-D-F-P-Q-L-C-Q-L-C-P-G-C-G-C-S-T-L-N-Q-Y-F-G-Y-S-G-A-F-K-C-L-K-D-G-A-G 201 D-V-A-F-V-K-H-S-T-I-F-E-N-L-A-N-K-A-D-R-D-Q-Y-E-L-L-C-L-D-N-T-R-K-P-V-D-E-Y-K-D-C-H-L-A-Q-V-P-S-H-T 251 V-V-A-R-S-M-G-G-K-E-D-L-I-W-E-L-L-N-Q-A-Q-E-H-F-G-K-D-K-S-K-E-F-Q-L-F-S-S-P-H-G-K-D-L-L-F-K-D-S-A-H ${ }^{301}$ G-F-L-K-V-P-P-R-M-D-A-K-M-Y-L-G-Y-E-Y-V-T-A-I-R-N-L-R-E-G-T-C-P-E-A-P-T-D-E-C-K-P-V-K-W-C-A-L-S-H-H. ${ }^{351}$ E-R-L-K-C-D-E-W-S-V-N-S-V-G-K-I-E-C-V-S-A-E-T-T-E-D-C-I-A-K-I-M-N-G-E-A-D-A-M-S-L-D-G-G-F-V-Y-I-A-G ${ }^{401}$ K-C-G-L-V-P-V-L-A-E-N-Y-N-K-S-D-N-C-E-D-T-P-E-A-G-Y-F-A-V-A-V-V-K-K-S-A-S-D-L-T-W-D-N-L-K-G-K-K-S-C. ${ }^{451}$ H-T-AV-G-R-T-A-GWN-I-P-MGL-L-Y-N-K-I-N-C-R-F-DE-F-F-S-EG-C-A-G-S-K-KDS-S-L-K-K-C-MG

501 S-G-L-N-L-C-E-P-N-N-K-E-G-Y-Y-G-Y-T-G-A-F-R-C-L-V-E-K-G-D-V-A-F-V-K-H-Q-T-V-P-Q-N-T-G-G-K-N-P-D-P-W

551 A-K-N-L-N-E-K-D-Y-E-L-L-C-L-D-G-T-R-K-P-V-E-E-Y-A-N-C-H-L-ALR-A-P-N-H-A-V-V-T-R-K-D-K-E-A-C-V-H-K-I

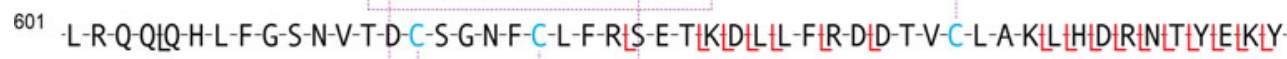

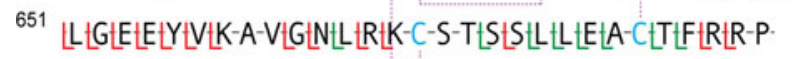




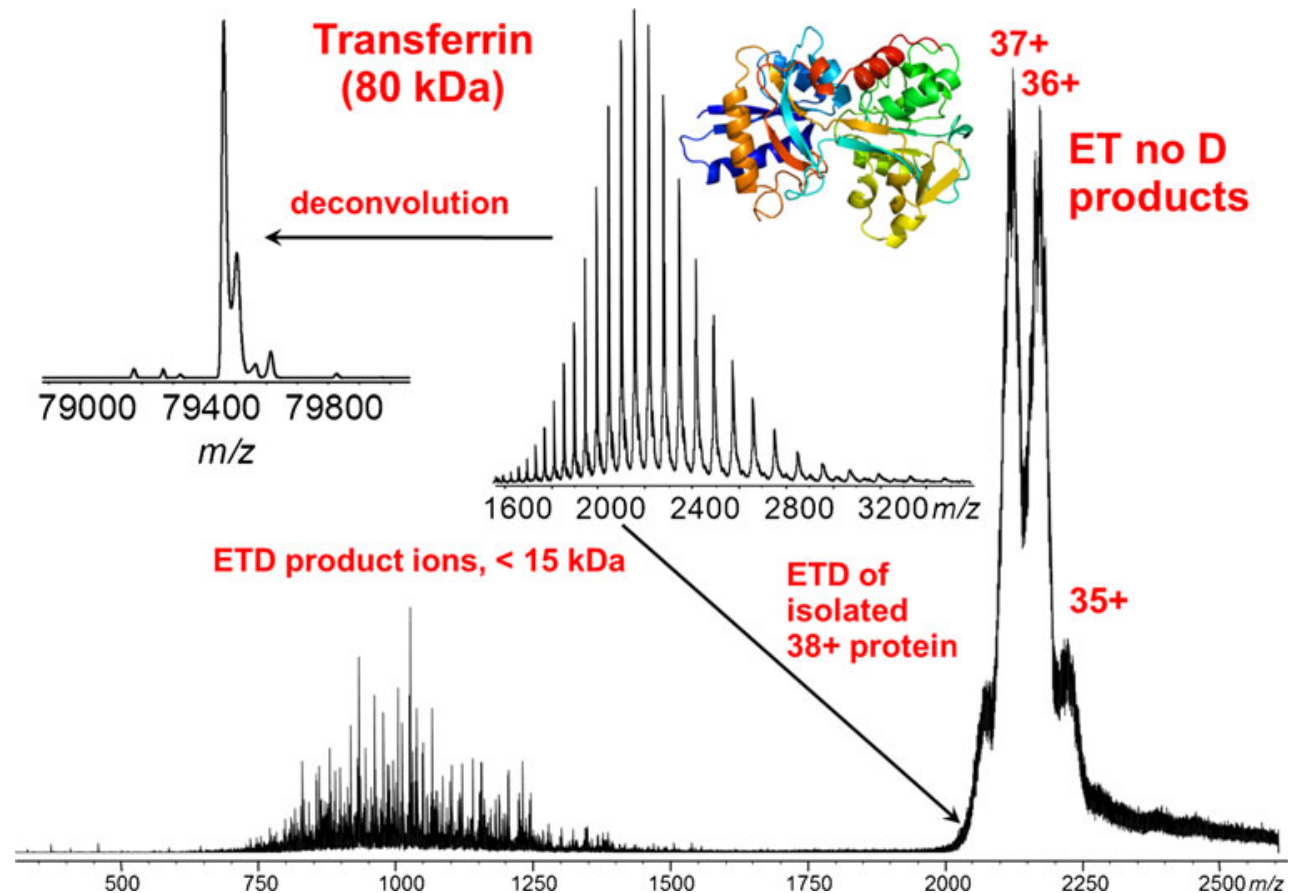

Fig. 4 Top-down electron transfer dissociation (ETD)-based ESI qTOF MS/MS of transferrin (79 kDa). The insets show ESI qTOF MS of intact transferrin with and without deconvolution. Prior to ETD, a single charge state, $[\mathrm{M}+38 \mathrm{H}]^{38+}$, of transferrin was selected with an isolation window of $30 \mathrm{~m} / \mathrm{z}$. Efficient charge reduction upon electron transfer resulted in electron transfer products without dissociation (ETnoD products), specifically $[\mathrm{M}+38 \mathrm{H}]^{37+\cdot},[\mathrm{M}+38 \mathrm{H}]^{36+\cdot \cdot}$, and $[\mathrm{M}+38 \mathrm{H}]^{35+\cdots \cdot}$ ions. Product ions are observed as a group primarily in the $m / z=700-1,600$ region. Due to their high charge state and lower abundance compared to the ETnoD charge-reduced precursor ions these ions are not resolved. There are no product ions detected above $2,500 \mathrm{~m} / \mathrm{z}$

formation of a significant product ion population without excessive internal fragment production, and incomplete dissociation of the charge-reduced species (Fig. S1, Electronic Supplementary Material). The radical anion reaction time can deeply influence the ETD efficiency as it determines the final reagent anion number involved in ETD. For a fixed ion-ion interaction time, a low anion injection time impedes ETD, and conversely at too long injection times the occurrence of multiple, consecutive electron transfer events is highly enhanced, so that many charge-reduced species, low-charged product ions and internal fragments are produced. The final applied value was $100 \mathrm{~ms}$ (Fig. S2, Electronic Supplementary Material). The ETD mass spectrum of oxidized transferrin is shown in Fig. 4, bottom. Charge-reduced species (particularly, $[\mathrm{M}+38 \mathrm{H}]^{37+*},[\mathrm{M}+38 \mathrm{H}]^{36+* \bullet}$, and $[\mathrm{M}+38 \mathrm{H}]^{35+\cdots}$ ions) dominated the mass spectrum in the high $\mathrm{m} / \mathrm{z}$ region, whereas the product ions were grouped and centered at $\sim 1,000 \mathrm{~m} / \mathrm{z}$. Notably, in contrast to what is typically observed for a smaller protein, e.g., carbonic anhydrase, the highly abundant ETnoD products were positioned above $2,000 \mathrm{~m} / \mathrm{z}$, thus they did not interfere with the product ion detection and analysis. Most product ions were located below $\sim 1,700 \mathrm{~m} / \mathrm{z}$.

An expanded view of the ETD mass spectrum of transferrin containing most of the product ions is presented in Fig. 5. The top panel illustrates the capabilities of the qTOF mass spectrometer to isotopically resolve multiply charged product ions 
Fig. 5 Expanded views on ETD product ions of transferrin obtained on a qTOF MS. (Bottom) expanded segment, $m / z=700-1,500$, shows ETD product ions that are assigned to be mainly $c$-type product ions of transferrin in charge states from $5+$ to $14+$. Product ions $c_{69}$ and $c_{56}$ are particularly abundant and present in a number of charge states. (Top) expanded segment, $m / z=975-1,015$, shows efficient separation and baseline resolution of multiply charged ETD product ions. For example, the inset shows a well-resolved isotopic distribution of $c_{55}{ }^{6+}$ product ion at $\sim 1,000 \mathrm{~m} / \mathrm{z}$. The annotated here MS/MS peaks are included into the protein sequence coverage summary presented in Scheme 1

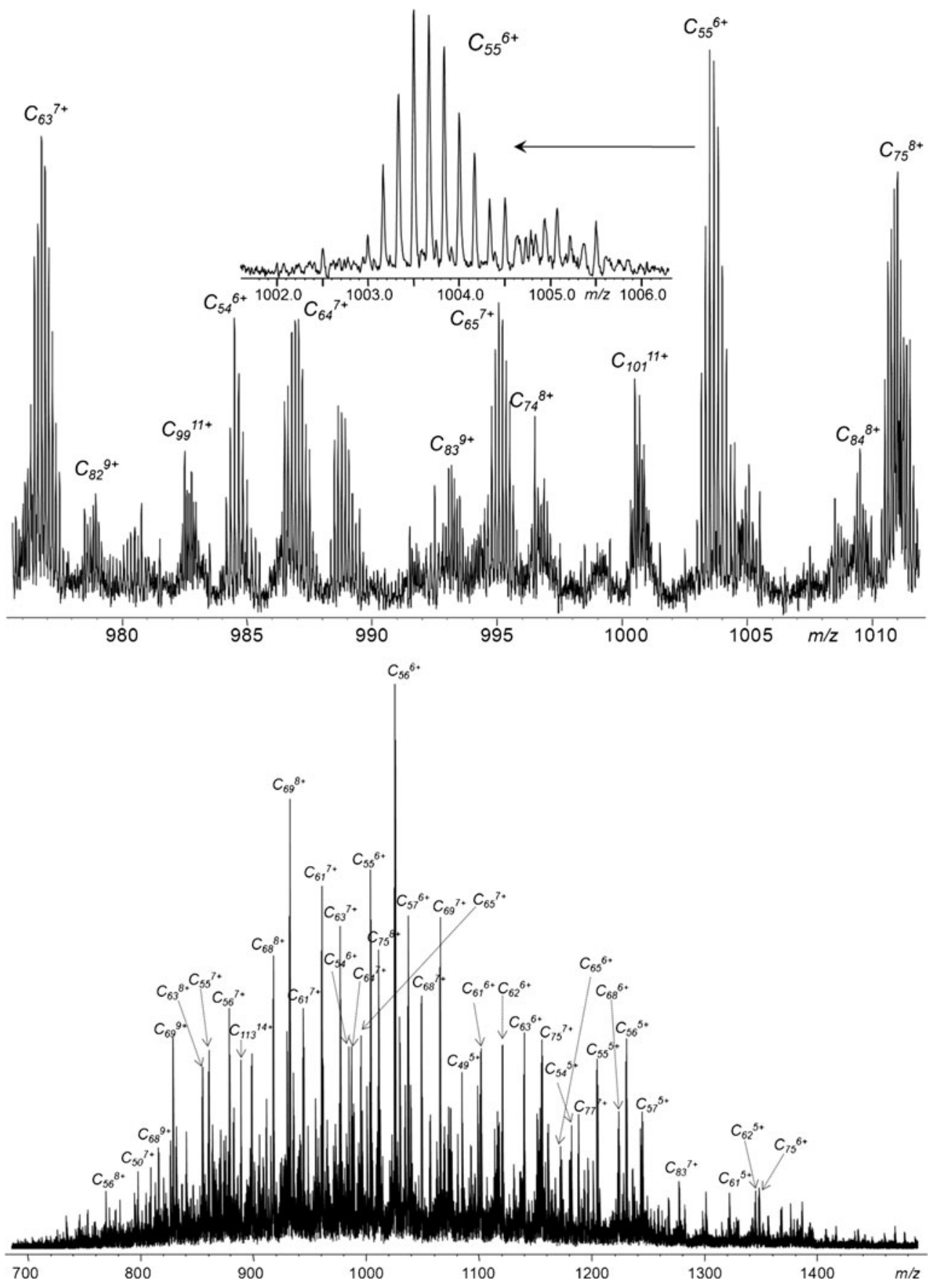

present in complex mixture. The assignment of the most abundant product ions shown in the bottom panel indicates that at the applied ETD conditions product ions up to $14^{+}$in charge state were detected, with charge states primarily between $5^{+}$and $8^{+}$. Almost all identified product ions were $c$-type, containing information about the $\mathrm{N}$-terminus of the protein. The relative position of the disulfide bridges may explain the fragmentation pattern observed. Close to the C-terminus, two Cys residues $\left(\mathrm{Cys}_{665}\right.$ and $\left.\mathrm{Cys}_{674}\right)$ are located. These are believed to be involved in the formation of distal S-S bridges with $\mathrm{Cys}_{474}$ and $\mathrm{Cys}_{402}$, respectively. The entire C-terminal portion of serotransferrin is folded in a tight conformation by a number of additional S-S bonds present between $\mathrm{Cys}_{402}$ and $\mathrm{Cys}_{674}$. The extent of disulfide-protected sequence is therefore most likely responsible for the formation of only few $z$-type ions. Particularly, most $z$-ions identified derived from backbone cleavages occurring at the $\mathrm{C}$-terminal side of $\mathrm{Cys}_{665}$ (i.e., with no need of cleaving a disulfide bond, but only the peptide bond for product ion formation), with the notable exceptions of $z_{18}, z_{75}$ and $z_{99}$.

Conversely, at the N-terminus there was a 69-residue loop completely disulfide-free (between $\mathrm{Ile}_{49}$ and $\mathrm{Ser}_{117}$ ), which 
interconnects the first disulfide-protected area (from Cys 9 to $\mathrm{Cys}_{48}$, with two S-S bonds) to the following one (starting from $\mathrm{Cys}_{118}$, which is linked to $\mathrm{Cys}_{194}$ ). As expected, most of the identified backbone cleavages arose from the unprotected linker, which was almost fully sequenced, and the small Nterminal sequence preceding $\mathrm{Cys}_{9}$. Only four cleavages in the first disulfide-protected area were identified (i.e., ions $c_{28}, c_{37}$, $c_{43}$, and $c_{44}$ ), and no product ions derived from the second area were observed. Overall, we identified $62 c$-ions and $11 z$-ions, for a total sequence coverage of $\sim 11 \%$ (Table 1 ).

The capability of ETD (and, more generally, radical-driven ion activation methods) to cleave disulfide bonds has been confirmed to some extent in these experiments with transferrin, in addition to those reported earlier for IgGs. Of 73 backbone cleavages reported for the oxidized serotransferrin, 13 derived from disulfide-protected regions, and were mainly located towards the C-terminus. However, recent results by Ganisl and Breuker suggest that, at least when applied to proteins, ECD does not preferentially nor frequently cleaves S-S bonds rather than peptide backbone ones [29], as reported in the past for peptides $[12,30]$. Nevertheless, the controversy may be limited to the term "preferential", as backbone cleavages from S-S bond-protected regions for many of the investigated proteins were reported. The authors explain these cleavages as resulting from a contribution from either vibrational fragmentation prior to ECD, or secondary radical fragmentation in ECD. Due to the differences in ETD and ECD processes, especially for what concerns the pressure of the ion traps where ion activation and fragmentation take place, further studies are needed to address this interesting question in relation specifically to ETD.

Top-down mass spectrometry of reduced transferrin Topdown experiment on disulfide-reduced serotransferrin was performed by direct infusion of a protein sample treated overnight with TCEP (Fig. 6). As expected, the reduction of $\mathrm{S}-\mathrm{S}$ bonds induced the exposure of protonation sites not accessible to the acidic solvent in the oxidized protein. As a result, the charge state envelope of the reduced intact protein is significantly shifted towards lower $\mathrm{m} / \mathrm{z}$ in respect to that of the oxidized counterpart. A large number of high charge state ions

Table 1 Summary of the assigned unique backbone cleavages for oxidized or reduced transferrin, and for the combination of the two datasets.

\begin{tabular}{lccl}
\hline $\begin{array}{l}\text { Serotransferrin } \\
\text { form }\end{array}$ & $\begin{array}{l}\text { N-terminal } \\
\text { fragments }\end{array}$ & $\begin{array}{l}\text { C-terminal } \\
\text { fragments }\end{array}$ & $\begin{array}{l}\text { Total sequence } \\
\text { coverage (\%) }\end{array}$ \\
\hline Oxidized & $62(4)^{\mathrm{a}}$ & $11(9)^{\mathrm{a}}$ & 10.7 \\
Reduced & 34 & 39 & 10.7 \\
Combined & 82 & 41 & 18.1 \\
\hline
\end{tabular}

${ }^{a}$ For the oxidized form, the number of backbone cleavages from disulfide-protected regions is indicated within parenthesis were detected (Fig. 6, right inset). Specifically, the charge state distribution included charge states from $40^{+}$to $100^{+}$, with the most intense transferrin ions located around $900 \mathrm{~m} / \mathrm{z}$ and carrying $83-88$ protons.

To maximize the fragmentation efficiency in radical-driven mass spectrometry, high charge state precursors are usually preferred. Nevertheless, positioning the precursor isolation window around $1,000 \mathrm{~m} / \mathrm{z}$ resulted in a partial overlapping between the ETnoD products and the heavy product ions. An intensity difference was observed between the chargereduced species and dissociation products. This impeded the identification of fragments above $1,000 \mathrm{~m} / \mathrm{z}$, therefore we opted for a $300 \mathrm{~m} / \mathrm{z}$ isolation window centered at $1,285 \mathrm{~m} / \mathrm{z}$, to include a number of charge states of the precursor (from $56^{+}$ to $70^{+}$) (Fig. S3, Electronic Supplementary Material). These precursor ions ensured improved fragmentation efficiency and the presence of ETnoD products in a region of the mass spectrum not occupied by product ions, beyond $1,400 \mathrm{~m} / \mathrm{z}$. Different from the oxidized transferrin, the ETD mass spectrum of the reduced protein showed a large $\mathrm{m} / \mathrm{z}$ distribution of the product ions, which were not grouped in a specific, limited region but instead spread between $\sim 200$ and $1,300 \mathrm{~m} / \mathrm{z}$, with a variation of intensity that generally is not higher than two- to three-folds over the whole $\mathrm{m} / \mathrm{z}$ range (Fig. 6, left insets). Since the same ETD fragmentation parameters (injection and interaction times) were applied for both the lower charge states oxidized, and higher charge state reduced forms, it is likely that for reduced transferrin analysis multiple electron transfer events occurred on already formed product ions, reducing their charge state and increasing their final mass-to-charge ratio. This is supported by the identification and assignment of several $z+1$ ions, generally not detected for the oxidized protein, and presumably obtained through a charge reduction event by secondary electron transfer on a primary product ion.

As summarized in Scheme 1, the distribution of the assigned product ions for ETD of reduced serotransferrin was more balanced between $c$ - and $z$-type ions. Regarding the N-terminal fragments, the backbone cleavages observed were complementary to those previously identified for the oxidized transferrin. Specifically, the protein motif comprised between $\mathrm{Cys}_{9}$ and $\mathrm{Cys}_{48}$ was highly sequenced (18/30 backbone cleavages assigned). On the contrary, the loop between $\mathrm{Cys}_{48}$ and $\mathrm{Cys}_{118}$ was poorly sequenced. The number of $z$ type ions was increased, with good sequence coverage in the region between $\mathrm{Cys}_{620}$ and the $\mathrm{C}$-terminus. This motif included also $\mathrm{Cys}_{637}$, which is linked to $\mathrm{Cys}_{418}$ in the oxidized protein; a non-complete reduction of this bond might explain the poor or absent sequence coverage for the sequence preceding $\mathrm{Cys}_{637}$. In general, for the reduced protein the average size of assigned product ions was smaller than for the oxidized counterpart, likely because of a modified conformation and charge location. Here, the only "high-mass" product ions assigned were $c_{101}, c_{102}$ and $c_{104}$ (which were still lighter than 


\section{ETD qTOF MS of reduced serotransferrin}

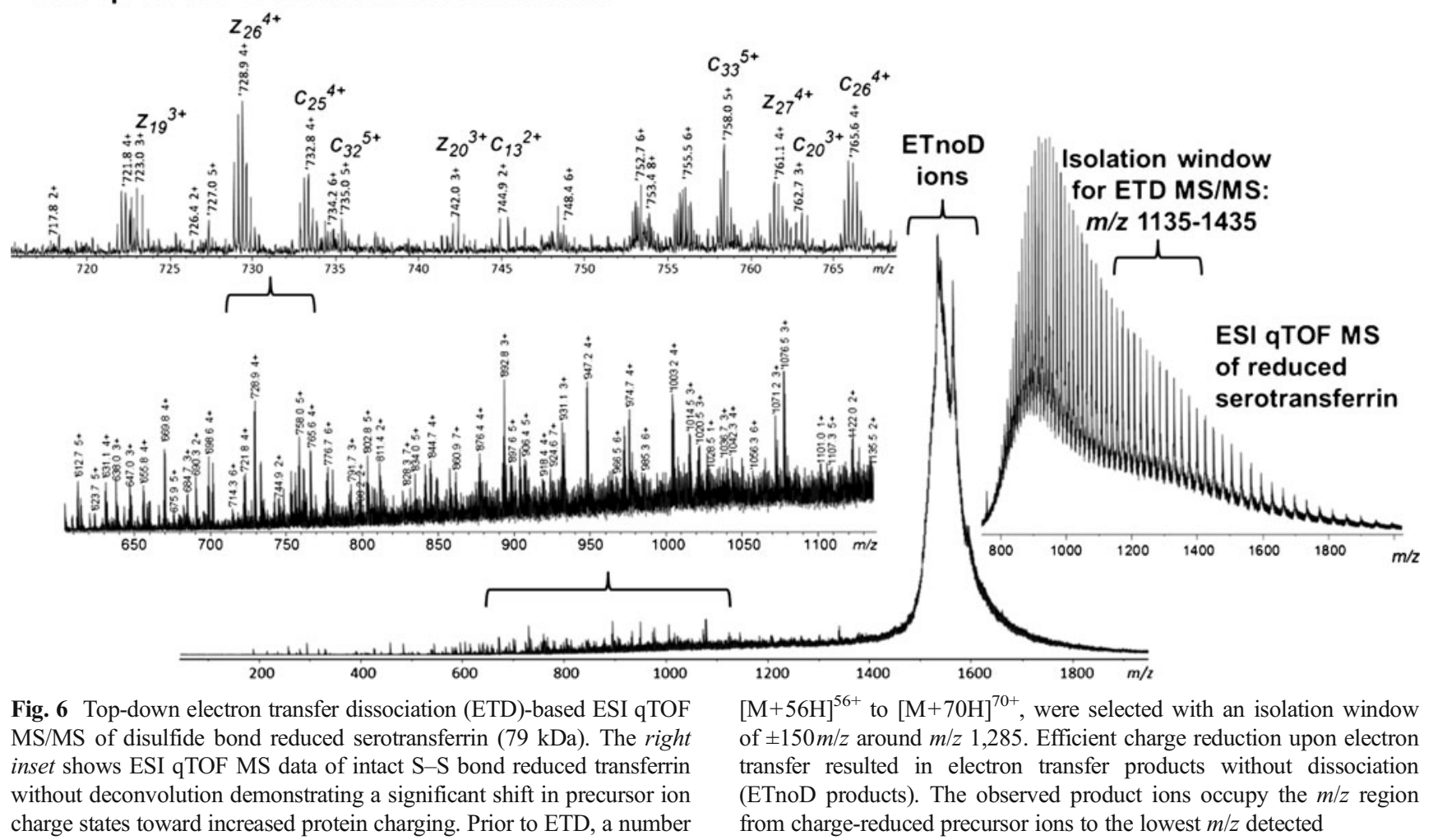

of transferrin precursor ions in different charge states, from about

other $c$-ions assigned for the oxidized transferrin). In total, for the reduced form we report $34 c$-type ions and $39 z$-type ions (Table 1). Similarly to the previous experiments on IgG fragmentation, no complementary fragments for the same backbone cleavage have been identified.

\section{Conclusions}

Our previous results reported for the analysis of immunoglobulin $\mathrm{G}$ (IgG) had already shown light on the new possibilities open by high-resolution TOF mass spectrometry for the topdown analysis of large proteins. Here, we focused our attention on electron transfer dissociation-based TD MS characterization, for stressing the current possibilities offered by this experimental set-up and further investigating the dependence of protein sequence coverage on the protein structure/conformation and its disulfide bond connectivity. The comparison between oxidized and reduced transferrin clarifies, despite the use of a different precursor charge state for the ETD reaction, that the presence or absence of $\mathrm{S}-\mathrm{S}$ bonds redistribute entirely the fragmentation channels (see Scheme 1, where the backbone cleavages in common between oxidized and reduced precursors, signed in green, are a minority). Not only the disulfideprotected regions need two bond cleavages to be sequenced, but also the different charge localization and local conformation can play an important role in this scenario. Moreover, the comparison between the fragmentation maps of transferrin and $\mathrm{IgG}$ represents incontrovertible evidence that ETD is primarily directed to the disulfide-free loops interconnecting consecutive disulfide-protected domains when the proteins are analyzed in non-reduced forms [27]. On the other hand, for these two protein forms the product ion populations are distributed very differently in their ETD mass spectra (a singlemaximum distribution for transferrin versus a doublemaximum distribution for the $\mathrm{IgG}$ ), indicating that also the primary structure of the protein influences the final outcome (see Fig. 1 and Schemes 1 and 2 in Ref. [27]). Serotransferrin presents a much more complicated S-S bond connectivity in comparison to the $\operatorname{IgG}$, and this might explain the limited sequence coverage for the $\mathrm{C}$-terminus of its oxidized form, as well as the smaller size of assigned fragments when compared to the IgG. The analysis of the reduced transferrin, indeed, complemented the results obtained for the oxidized form very well: although the extent of fragmentation obtained in each single experiment (i.e., for the oxidized or reduced protein) is the same, the combination of the two increases the final sequence coverage by $\sim 70 \%$ (from 10.7 to $18.1 \%$, Table 1 ).

Achieved ETD TD MS performance shall extend the application range of protein-drug binding characterization to larger proteins, compared to the current level of ubiquitin $(8.6 \mathrm{kDa})$ size proteins $[31,32]$. However, further enhancement in (1) 
sensitivity of detection of multiply charged product ions (to reach high signal-to-noise by averaging a lower number of scans); (2) dissociation of charge-reduced species, which are constantly dominant over the product ions in all the reported mass spectra; (3) detection of complementary heavy product ions as well as (4) internal product ions from secondary fragmentation will be needed to increase the overall capabilities of ETD-based TD MS and TDP on the qTOF and alternative, e.g., Orbitrap FTMS, platforms.

Acknowledgments Ünige Laskay and Michael Groessl are gratefully acknowledged for motivating discussions. We also thank Anja Resemann for the kind assistance in data analysis. This work was supported by the Swiss National Science Foundation (SNF project 200021-125147/1).

\section{References}

1. Cui W, Rohrs HW, Gross ML (2011) Analyst 136:3854-3864

2. Tran JC et al (2011) Nature 480:254-258

3. Tipton JD, Tran JC, Catherman AD, Ahlf DR, Durbin KR, Kelleher NL (2011) J Biol Chem 286:25451-25458

4. Smith LM, Kelleher NL, Consortium for Top Down Proteomics (2013) Nat Methods 10:186-187

5. Tran JC, Doucette AA (2008) Anal Chem 80:1568-1573

6. Zheng S, Yoo C, Delmotte N, Miller FR, Huber CG, Lubman DM (2006) Anal Chem 78:5198-5204

7. Zubarev RA, Kelleher NL, McLafferty FW (1998) J Am Chem Soc 120:3265-3266

8. Zubarev RA, Horn DM, Fridriksson EK, Kelleher NL, Kruger NA, Lewis MA, Carpenter BK, McLafferty FW (2000) Anal Chem $72: 563-573$

9. Syka JEP, Coon JJ, Schroeder MJ, Shabanowitz J, Hunt DF (2004) P Natl Acad Sci USA 101:9528-9533

10. Wells JM, McLuckey SA (2005) Methods Enzymol 402:148-185

11. Little DP, Speir JP, Senko MW, O'Connor PB, McLafferty FW (1994) Anal Chem 66:2809-2815
12. Zubarev RA, Kruger NA, Fridriksson EK, Lewis MA, Horn DM, Carpenter BK, McLafferty FW (1999) J Am Chem Soc 121:28572862

13. Shaw JB, Li W, Holden DD, Zhang Y, Griep-Raming J, Fellers RT, Early BP, Thomas PM, Kelleher NL, Brodbelt JS (2013) J Am Chem Soc doi:10.1021/ja4029654

14. Zubarev RA (2003) Mass Spectrom Rev 22:57-77

15. Silivra OA, Kjeldsen F, Ivonin IA, Zubarev RA (2005) J Am Soc Mass Spectrom 16:22-27

16. Cooper HJ, Hakansson K, Marshall AG (2005) Mass Spectrom Rev 24:201-222

17. Sze SK, Ge Y, Oh H, McLafferty FW (2002) Proc Natl Acad Sci USA 99:1774-1779

18. Tsybin YO, Ramstrom M, Witt M, Baykut G, Hakansson P (2004) J Mass Spectrom 39:719-729

19. Ge Y, Lawhorn BG, ElNaggar M, Strauss E, Park JH, Begley TP, McLafferty FW (2002) J Am Chem Soc 124:672-678

20. Baba T, Hashimoto Y, Hasegawa H, Hirabayashi A, Waki I (2004) Anal Chem 76:4263-4266

21. Satake H, Hasegawa H, Hirabayashi A, Hashimoto Y, Baba T, Masuda K (2007) Anal Chem 79:8755-8761

22. Syka JE, Coon JJ, Schroeder MJ, Shabanowitz J, Hunt DF (2004) Proc Natl Acad Sci USA 101:9528-9533

23. Huang TY, McLuckey SA (2010) Proteomics 10:3577-3588

24. Hartmer RG, Kaplan DA, Stoermer C, Lubeck M, Park MA (2009) Rapid Commun Mass Spectrom 23:2273-2282

25. McAlister GC, Phanstiel D, Good DM, Berggren WT, Coon JJ (2007) Anal Chem 79:3525-3534

26. Kaplan DA, Hartmer R, Speir JP, Stoermer C, Gumerov D, Easterling ML, Brekenfeld A, Kim T, Laukien F, Park MA (2008) Rapid Commun Mass Spectrom 22:271-278

27. Tsybin YO, Fornelli L, Stoermer C, Luebeck M, Parra J, Nallet S, Wurm FM, Hartmer R (2011) Anal Chem 83:8919-8927

28. Fornelli L, Damoc E, Thomas PM, Kelleher NL, Aizikov K, Denisov E, Makarov A, Tsybin YO (2012) Mol Cell Proteomics 11:1758-1767

29. Ganisl B, Breuker K (2012) ChemistryOpen 1:260-268

30. Gunawardena HP, Gorenstein L, Erickson DE, Xia Y, McLuckey SA (2007) Int J Mass Spectrom 265:130-138

31. Hartinger CG, Tsybin YO, Fuchser J, Dyson PJ (2008) Inorg Chem 47:17-19

32. Meier SM, Tsybin YO, Dyson PJ, Keppler BK, Hartinger CG (2012) Anal Bioanal Chem 402:2655-2662 